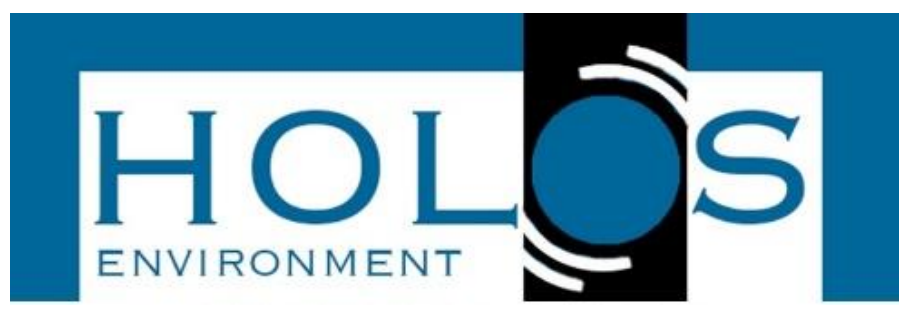

\title{
PERCEPÇÃO E CONHECIMENTO DE ALUNOS DE GRADUAÇÃO E FUNCIONÁRIOS DE UMA IES EM RELAÇÃO ÀS ESPÉCIES VEGETAIS E ÁREAS NATURAIS DO CAMPUS
}

\section{PERCEPTION AND KNOWLEDGE OF COLLEGE STUDENTS AND STAFF OF THE HIGHER EDUCATION INSTITUTION REGARDING CAMPUS PLANT SPECIES AND NATURAL AREAS}

\author{
Marina Consuli Tischer ${ }^{1}$; Alex Fernando Carciragui ${ }^{1}$; Andressa Cristina Ricardo \\ Camargo ${ }^{1}$;Lorena Laís Sala ${ }^{1}$; Natália de Souza Penteado'; \\ Tatiane Aparecida Domingues da Silva ${ }^{1}$;Giovana de Oliveira Pereira ${ }^{1}$; Jéssica Fernanda \\ Pupi ${ }^{1}$; Maiara Fernanda Cardoso ${ }^{1}$; Sheron G. Dias D'Ávilla ${ }^{1}$; Gabriele Marcon Oliveira'; \\ Maria Júlia N. Fernandes ${ }^{1}$; Angel Domiciano Santanna Santos ${ }^{1}$
}

Artigo recebido em: 22/11/2019 e aceito para publicação em: 08/01/2020.

DOI: http://dx.doi.org/10.14295/holos.v20i1.12359

\begin{abstract}
Resumo: As áreas verdes urbanas são percebidas de diferentes formas pelas pessoas, a depender de fatores culturais, sociais e sensoriais. Uma Instituição de Ensino Superior (IES) reunindo uma heterogeneidade de classes sociais e descendências étnicas torna-se um local de pesquisa que pode refletir diferentes percepções ambientais. O conhecimento de tal heterogeneidade serve de base para a construção de programas específicos de educação ambiental. Desta forma, o objetivo deste trabalho foi analisar a percepção e conhecimento de alunos de graduação presencial e funcionários da IES em relação às espécies de flora e áreas naturais do campus universitário. O método adotado foi a aplicação de entrevistas estruturadas contando questões abertas e fechadas. Os dados foram analisados quali e quantitativas. Foram 40 participantes, sendo $55 \%$ alunos e $45 \%$ funcionários. O número de espécies citadas pelos participantes variou entre 1 e 11 ( \pm 2.93$)$. Sobre as áreas naturais do campus a maioria dos entrevistados (73\%) a declarou como "regular" segundo sua percepção, sendo alguma forma de utilidade ao homem as mais citadas, ficando as funções ecológicas com menor importância. Baseando-se no conceito da "cegueira botânica" os resultados mostram que os entrevistados apresentam dificuldade em perceber as plantas em seu cotidiano. Tendo a referida IES a divulgação de uma postura sustentável, esperamos que os resultados possam servir de base para o planejamento de projetos de Educação Ambiental envolvendo alunos e funcionários da instituição e comunidade externa em projetos de extensão universitária que contribuam para o alcance de uma comunidade acadêmica que contribua com seu pilar ambiental.
\end{abstract}

Palavras-chave: Etnobotânica. Universitários. Percepção ambiental.

\begin{abstract}
Urban green areas are perceived in different ways by people, depending on cultural, social and sensory factors .A Higher Education Institution (HEI) bringing together a heterogeneity of cultures becomes a research place that may reflect different environmental perceptions. Knowledge of such heterogeneity support the construction of specific environmental education programs. Thus, the objective of this work was to analyze the perception and knowledge of college students and HEI staff regarding the species of flora and natural areas of the university campus. The method adopted was the application of structured interviews with open

\footnotetext{
1 Centro Universitário da Fundação Hermínio Ometto (FHO). Araras, SP E-mails: (mctbio@yahoo.com.br, carciragui@gmail.com, andressarajaram@gmail.com, lorenalaissala@gmail.com, natysouzapenteado@gmail.com, tati.domingues.silva@gmail.com, giovana.oliveirap@gmail.com, jesspupi@hotmail.com, maiarafc@hotmail.com, she dias@hotmail.com, gabrielemarcon@hotmail.com, maju.nfernandes@hotmail.com, angel.santanna09@gmail.com)
} 
and closed questions. The data were analyzed qualitatively and quantitative. There were 40 participants, $55 \%$ students and $45 \%$ staff. The number of species cited by participants ranged from 1 to 11 ( $\pm 2.95, S D 2.93)$. Regarding the natural areas of the campus, the majority of respondents (73\%) declared it as "regular" according to their perception, being some form of utility to people the most cited, with the ecological functions being less important. Based on the concept of "plant blindness" the results show that the interviewees have difficulty in understanding the plants in their daily lives. Having said a sustainable posture, we hope that the results can serve as a basis for the planning of Environmental Education projects involving students and staff of the institution and external community in university extension projects that contribute to the reach of an academic community that contributes to its environmental pillar.

Keywords: Ethnobotany. College students. Environmental perception.

\section{INTRODUÇÃO}

Áreas verdes urbanas tendem a ser valorizadas pela população por seus benefícios ao bem-estar diário das pessoas (LOBODA e ANGELIS 2005), no entanto, nem sempre são verdadeiramente reconhecidas e exploradas. Compreender tais relações de pessoas com recursos e elementos vegetais é campo de pesquisa das ciências da Etnobiologia e Etnobotânica (ALBUQUERQUE et al., 2009) e envolve dois conceitos-chave: a percepção ambiental e a "cegueira botânica" (TUAN, 2012; KUHNEN e HIGUCHI, 2011; WANDERSEE e SCHUSSLER, 2001).

A percepção ambiental envolve diversos fatores, entre eles, valores culturais e sociais, percepção sensorial e conhecimento sobre o mundo natural a nossa volta (SILVA e ALBUQUERQUE, 2014). Tais fatores estão inseridos no fenômeno introduzido por Wandersee e Schussler (2001) chamado por "cegueira botânica" ("plant blindness"). O termo foi criado com o intuito de conceituar à falta de habilidade das pessoas em perceber o "verde" ou espécies vegetais à sua volta. Uma pluralidade de causas é discutida e a falha na capacidade de perceber a importância e trabalhar a favor da conservação é citada como uma das consequências mais preocupantes.

Os espaços urbanos são um conjunto de elementos antrópicos e ambientais moldados e combinados pelas mãos do homem (LOBODA e ANGELIS 2005). Assim, as espécies vegetais presentes em uma área refletem, entre outras coisas, a visão utilitarista que podemos ter do meio ambiente (ALBUQUERQUE et al., 2019). Todavia, morar em uma área urbana ou em uma área rural traz percepções e vivências diferentes em relação ao meio natural a sua volta.

Em uma instituição de ensino superior (IES) é possível encontrar uma heterogeneidade de classes sociais e heranças étnicas que refletem diferentes comportamentos e percepções, tornando-se desta forma um local de pesquisa que pode mostrar heterogeneidades importantes em relação à percepção ambiental (WACHHOLZ, 2013). O trabalho de Carvalho et al. (2012) mostrou que estudantes de uma universidade em Sergipe acreditam que a arborização contribui de forma positiva para o rendimento estudantil, mas ao mesmo tempo mostrou a necessidade de intervenção de projetos de Educação Ambiental para integrar cursos que não sejam da área ambiental com o ambiente natural do campus. 
Diante do exposto, o presente estudo teve o objetivo de analisar a percepção e conhecimento de alunos de graduação presencial e funcionários da IES em relação às espécies de flora e áreas naturais do campus universitário.

\section{MATERIAL E MÉTODOS}

A pesquisa foi realizada no Centro Universitário da Fundação Hermínio Ometto FHO (área total $392.508 \mathrm{~m}^{2}$, área construída 50.063,61 m²) (Figura 1), localizado na cidade de Araras/SP. A cidade está inserida em uma área de vegetação característica de Mata Atlântica, com fitofisionomias de Floresta Estacional Semidecidual, florestas secundárias, vegetação ripária e enclaves de Cerrado (FADEL et al., 2012). A instituição tem o curso de Ciências Biológicas como um dos mais tradicionais, portanto, a transformação do campus, principalmente para o aumento de sua área natural tem sido constante desde sua fundação, onde a plantação de mudas de plantas nativas é prioridade.

Figura 1 - Campus do Cento Universitário da Fundação Hermínio Ometto - FHO. Fonte da imagem: Centro Universitário da Fundação Hermínio Ometto - FHO - site institucional.

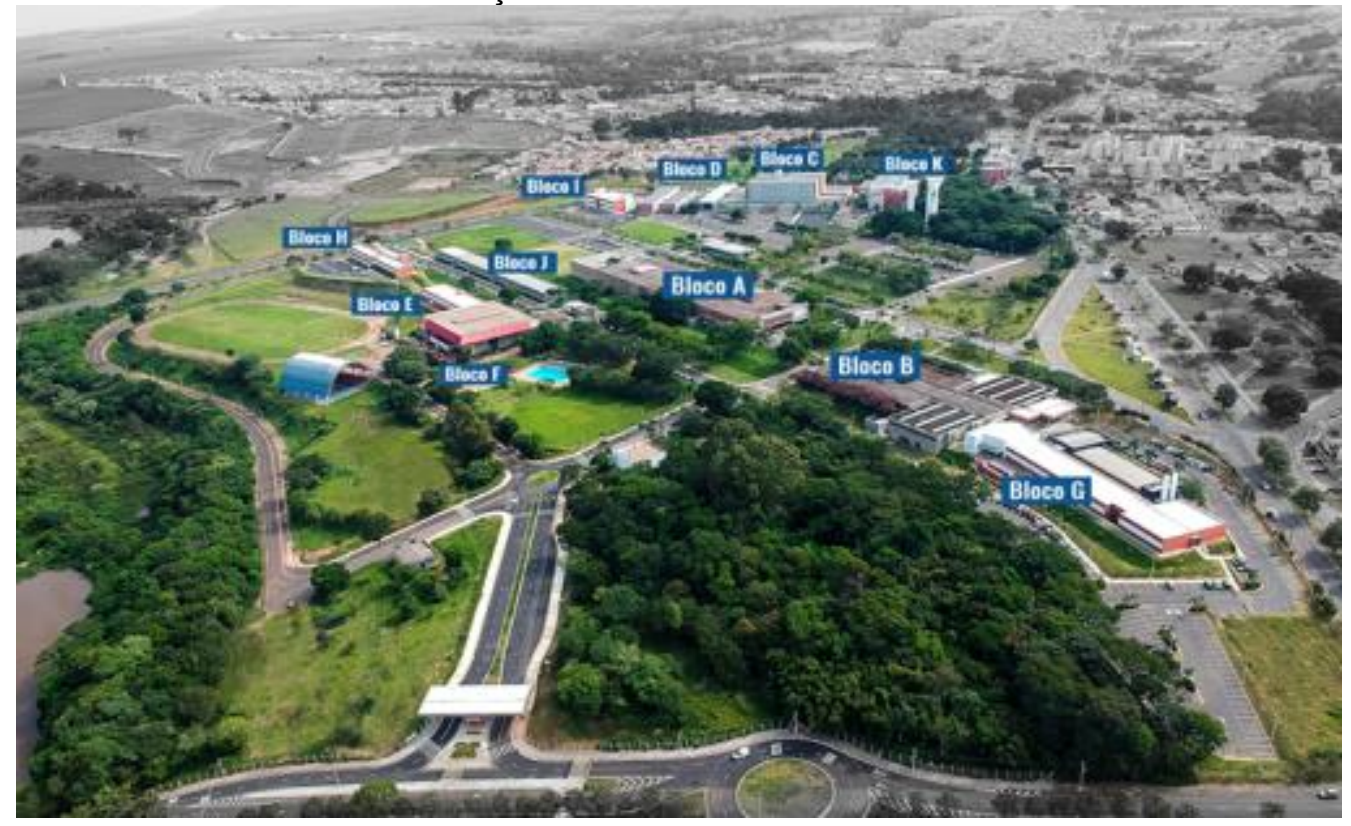

A coleta de dados foi através da aplicação de entrevistas a estudantes de graduação presencial fora do seu período regular de aula e funcionários da IES. O roteiro de entrevista estruturado (ALBUQUERQUE et al., 2010) foi montado a partir de pesquisa bibliográfica prévia e experiências pessoais e conhecimento dos autores e equipe deste estudo. Após validação em projeto piloto (DITT et al., 2003) o roteiro de entrevista foi estruturado em duas partes: 1 - Informações sócio-econômicas do participante e 2 - Conhecimento sobre espécies de plantas e áreas naturais do campus. O roteiro continha questões abertas e fechadas.

Buscou-se abranger na coleta de dados alunos de todas as áreas de ensino deste Centro Universitário, que estão divididas em: saúde, negócios, educação e engenharias e tecnologias e funcionários de diversos setores que realizam suas atividades tanto na área 
interna como externa do campus. A coleta de dados aconteceu entre os meses de novembro e dezembro de 2018.

Esta pesquisa foi aprovada pelo Comitê de Ética em Pesquisa sob autorização CAAE 10666918.7.0000.5385 e todos os participantes receberam o Termo de Consentimento Livre e Esclarecido (TCLE) concordando em participar da pesquisa.

Os dados foram analisados qualitativamente e quantitativamente, assim, toda informação pertinente ao assunto pôde ser analisada. As respostas das questões abertas foram categorizadas para análise.

\section{RESULTADOS E DISCUSSÃO}

Neste estudo, totalizou-se 40 participantes, sendo $55 \%$ alunos e $45 \%$ funcionários, com idades variando de 18 a 70 anos $( \pm 14.24)$ (Tabela 1$)$. Apenas 1 participante declarou ser morador de área rural, portanto a variável "moradia" não pôde ser analisada de forma mais detalhada.

Tabela 1 - Perfil sócio-econômico dos entrevistados

\begin{tabular}{ll}
\multicolumn{1}{c}{ Alunos } & $n$ \\
Sexo & \\
Homens & 12 \\
Mulheres & 10 \\
Moradia & \\
área urbana & 21 \\
área rural & 1 \\
Idade & \\
Mínimo & \\
Máximo & 18 \\
média & 24 \\
dp & 20,3 \\
\multicolumn{1}{c}{ Funcionários } & \\
Sexo & 1,7 \\
Homens & \\
Mulheres & 7 \\
Moradia & 11 \\
área urbana & 16 \\
área rural & 0 \\
Idade & \\
Mínimo & \\
Máximo & \\
média \\
dp
\end{tabular}


Quando perguntados sobre se e quantas espécies de plantas do campus conheciam, os valores variaram entre 1 e $11( \pm 2.95, \mathrm{dp} 2.93)$ e 8 participantes declararam não conhecer nenhuma das espécies de flora presentes. Participantes que citaram acima de cinco espécies de plantas foram em sua maioria funcionários $(n=8)$, enquanto que alunos, que tendem a passar menos tempo no campus, apenas dois citaram mais de cinco espécies, sendo estes do curso de Ciências Biológicas e Farmácia, que em seu conteúdo pedagógico possuem contato maior as plantas. A baixa percepção quanto ao número de espécies também pode ser resultado à expressividade que algumas espécies arbóreas de grande porte exercem no campo visual, sendo mais facilmente observadas e assim "escondendo" espécies menores.

Os grupos de plantas mais citados foram espécies arbóreas ( $n=27$ participantes) e plantas medicinais ( $n=8$ participantes).

Quando perguntados sobre as áreas naturais do campus $8 \%(n=3)$ declaram ser "pouca", enquanto 20\% ( $n=8)$ declararam como "muita" e 73\% como "regular" ( $n=29)$. O estudo de Assunção et al. (2017) sobre a percepção ambiental e de transformação de um campus universitário e seu entorno, mostra que a supressão de áreas naturais para expansão urbana, nem sempre é vista de forma negativa. A expansão de áreas urbanas e a construção de prédios dá às pessoas a noção de desenvolvimento, o que muitas vezes não é associado como compatível com preservação ambiental.

Além das áreas verdes presentes nos prédios e estacionamento, o campus possui algumas outras áreas e instalações destinadas às áreas naturais. São elas: arboreto, canteiro de plantas medicinais, arena e córrego do Andrézinho. Os entrevistados prioritariamente relataram conhecer todas estas outras áreas (Figura 2). Estas áreas não são usadas ou frequentadas no dia-a-dia pelos alunos ou funcionários da instituição, no entanto algumas são utilizadas para eventos institucionais, sendo este o motivo do alto índice de conhecimento pelos participantes.

Excluindo-se os funcionários das áreas de manutenção e vigilância, que frequentas estas áreas devido às suas atividades de trabalho, 59\% $(n=19)$ dos entrevistados declaram ter conhecido ou utilizado estas áreas para lazer, enquanto $41 \%(n=13)$ declaram terem ido por alguma atividade relacionada ao curso ou evento institucional.

Quanto à função das áreas naturais do campus, alguma forma de utilidade ao homem foi citada por 26 participantes, enquanto as funções relacionadas à estética $(n=20)$ e à ecologia $(n=8)$ tiveram menos citações (Tabela 2). 
Figura 2 - Conhecimento dos participantes em relação às instalações e áreas naturais do campus
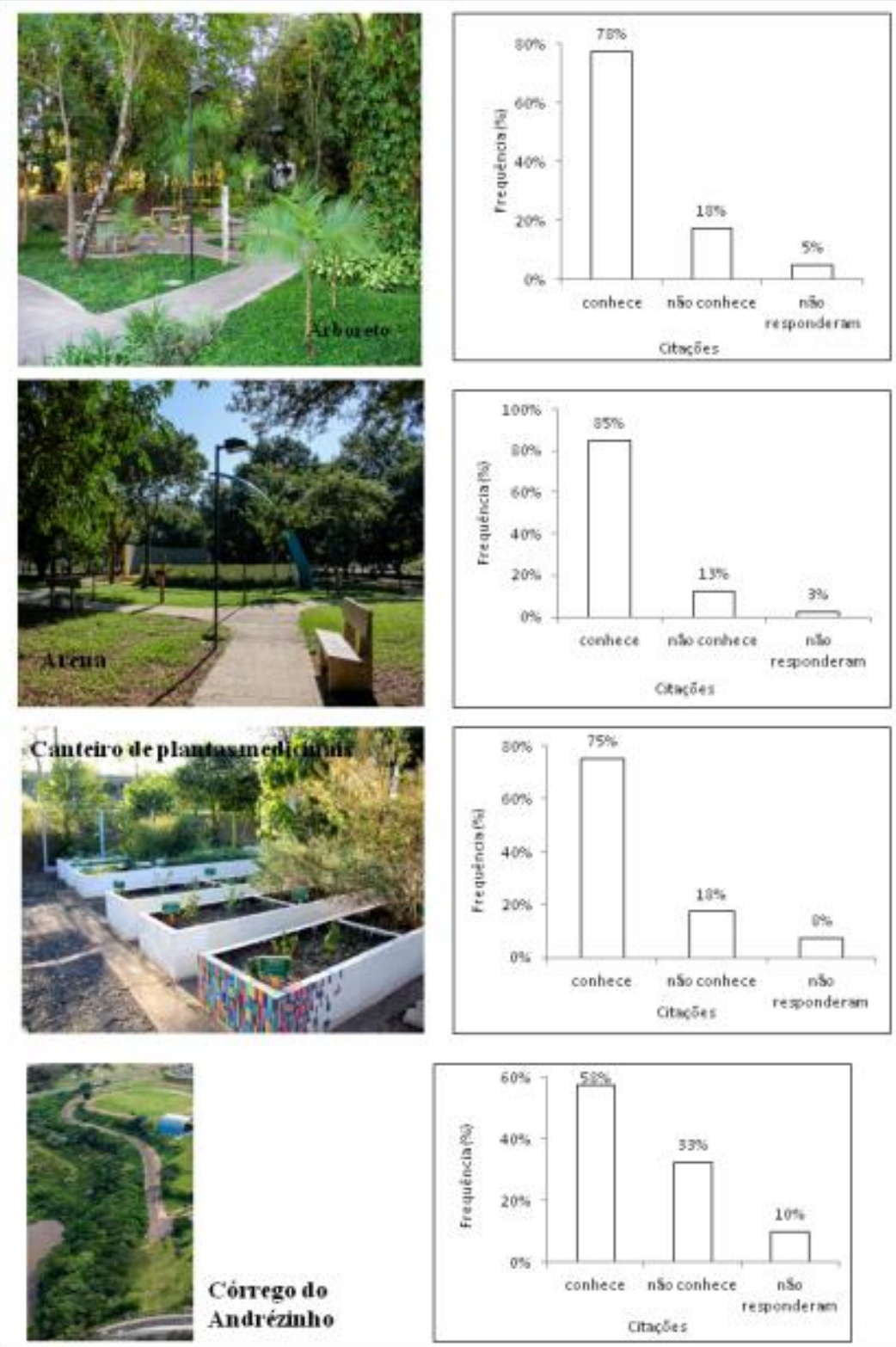

Fonte: Centro Universitário da Fundação Hermínio Ometto - FHO - site institucional

Tabela 2 - Exemplos de citações categorizadas para a questão: "Na sua opinião, qual a importância da área naturais do campus"?

\section{Utilidade ao homem}

"Ajuda no ambiente do seu trabalho, deixa o ar mais fresco"

"Harmonia e ventilação"

\section{Estética}

"Beleza do local"

"Compor a arquitetura"

\section{Ecológica}

"Atrais as aves"

"É essencial ao meio ambiente" 
As análises dos dados e resultados desta pesquisa devem ser melhor desenvolvidas posteriormente, no entanto, esperamos que possam contribuir com o planejamento de novos projetos envolvendo as áreas naturais do campus pela instituição. Os resultados podem também servir de base para o planejamento de projetos de Educação Ambiental envolvendo alunos e funcionários da instituição e comunidade externa em projetos de extensão universitária.

Baseando-se no conceito da "cegueira botânica" de Wandersee e Schussler (2001) os resultados mostram que relacionado à percepção, os entrevistados apresentam dificuldade em perceber as plantas em seu cotidiano e prioritariamente não percebem suas características como adaptações, diversidade, perfume, etc. Acerca do conhecimento, não sabem explicar o básico sobre as plantas da sua região, uma característica apontada pelo trabalho de Wandersee e Schussler (2001).

Na mesma IES, Tischer et al. (submetido à publicação) pesquisaram sobre a relação dos estudantes com animais não-domésticos presentes no campus e registraram um número menor de citações de espécies animais por participante $( \pm 1.62)$, comparado com as citações de espécies vegetais nesta pesquisa. Este ponto registra desacordo com um dos preceitos do trabalho de Wandersee e Schussler (2001), que discute sobre a prevalência de percepção das espécies animais em relação aos vegetais.

\section{CONCLUSÃO}

Em função da tradição do curso de Ciências Biológicas e da postura de seus fundadores, a referida IES mostra postura sustentável e a favor do aumento e melhoria das áreas naturais do campus. No entanto, vê-se a necessidade de mostrar aos seus alunos e funcionários a importância desta postura ambiental através de programas de Educação Ambiental que os informe sobre as espécies presentes no campus e a importância de suas presenças no aspecto de um ambiente urbano ecologicamente enriquecido e no aspecto da melhoria do bem-estar humano. O aumento da inclusão das áreas naturais do campus em atividades dos cursos ou eventos institucionais pode aumentar o interesse da comunidade acadêmica pelas áreas naturais do campus.

Ações como o destaque de algumas espécies, com placas informativas contendo os nomes populares e científico e informações básicas sobre a espécie podem ser adotadas. Uma exposição de fotos sobre o tema e/ou a produção de material impresso ou videográfico pode ser produzida. Desta forma, a comunidade acadêmica poderia ser informada ao mesmo tempo que pode-se fazer a valorização dos profissionais que fazem a manutenção das áreas verdes do campus e de seus conhecimentos populares.

\section{AGRADECIMENTOS}

Agradecemos ao Centro Universitário da Fundação Hermínio Ometto - FHO, através da coordenação do curso de Ciências Biológicas, da Pró-reitoria de Graduação e da Próreitoria de Comunidade e Extensão pela disposição em apoiar e participar da pesquisa. 


\section{REFERÊNCIAS}

ALBUQUERQUE, U. P.; ARAÚJO, T. A. S.; RAMOS, M. A.; NASCIMENTO, V. T.; LUCENA, R. F. P.; MONTEIRO, J. M.; ALENCAR, N. L.; ARAÚJO, E. L. How ethnobotany can aid biodiversity conservation: reflections on investigations in ihe semi-arid region of NE Brazil. Biodivers Conserv. v. 18, n. 1, p. 127-150, 2009. doi: 10.1007/s10531-008-9463-8. https://doi.org/10.1007/s10531-008-9463-8

ALBUQUERQUE, U. P.; LUCENA, R. F. P.; ALENCAR, N. L. Métodos e técnicas para coleta de dados etnobiológicos. In: ALBUQUERQUE, U. P.; LUCENA, R. F. P.; CUNHA, L. V. F. C. (orgs). Métodos e técnicas na pesquisa etnobiológica e etnoecológica. São Paulo (Brasil): Nupeea; 2010. p. 41-54.

ALBUQUERQUE, U. P.; MEDEIROS, P. M.; FERREIRA-JUNIOR, W. S.; SILVA, T. C.; SILVA R. R. V.; SOUZA, T. G. Social-ecological theory of maximization: basic concepts and two initial models. Biol Theo. v. 14, n. 2, p. 73-85, 2019. doi: 10.1007/s13752-019-00316-8. https://doi.org/10.1007/s13752-019-00316-8

ASSUNÇÃO, A. F.; GUISLON, A. V.; ARRUDA, D. B.; VIRTUOSO, J. C.; BÔLLA, K. D. S.; CORREAA, P. F.; PADILHA, P. T.; ASSUNÇÃO, V. K. Transformações ambientais de um campus universitário nas últimas três décadas: um estudo baseado na percepção ambiental de atores da instituição. Revis Esp. v. 38, n. 23, p. 17, 2017.

CARVALHO, R. F.; OLIVEIRA, L. A. R.; FRANÇA, T. P. P.; RAMO, L. S. Percepção dos alunos quanto a arborização da UFS-Campus de São Cristóvão. Sci Plena. v. 8, n. 4, p. 1-7, 2012.

DITT, E. H.; MANTOVANI, W.; VALLADARES-PADUA, C.; BASSI, C. Entrevistas e aplicação de questionários em trabalhos de conservação. Métodos de estudos em Biologia da Conservação e Manejo da Vida Silvestre. Curitiba: Editora da Universidade Federal do Paraná, p. 631-646, 2003.

FADEL, N.; RAYMUNDO-JUNIOR, O.; SAYEG, H. S. Caracterização e avaliação temporal de remanescentes florestais do município de Araras/São Paulo. Hol Environ. v. 12, n. 2, p. 215-224, 2012. doi: 10.14295/holos.v12i2.4687. https://doi.org/10.14295/holos.v12i2.4687

KUHNEN, A.; HIGUCHI, M. I. G. Percepção ambiental. In: CAVALCANTE, S.; ELALI, G. A. (orgs). Temas Básicos em Psicologia Ambiental. Petrópolis (Brasil): Editora Vozes. 2011.

LOBODA, C. R.; ANGELIS, B. L. D. De. Áreas verdes públicas urbanas: conceitos, usos e funções. Revis Ambi. v. 1, n. 1, p. 125-139.

SILVIA, T. C.; ALBUQUERQUE, U. P. O que é percepção ambiental? In: ALBUQUERQUE, U. P. (org.). Introdução à Etnobiologia. Recife (Brasil): Nupeea. 2014. p. 55-58.

TUAN, Y. Topofilia: um estudo da percepção, atitudes e valores do meio ambiente. São Paulo (Brasil): Editora Difel. 2012.

WACHHOLZ, C. B. Educação, natureza e sustentabilidade: a percepção da paisagem no campus da PUCRS. 2013. Dissertação (Mestrado em Educação) - Pontifícia Universidade Católica do Rio Grande do Sul, Porto Alegre.

WANDERSEE, J. H. SCHUSSLER, E. E. Toward a theory of plant blindness. Plant Science Bulletin. v. 47, n. 1, p. 2-9, 2001. doi: 10.2307/4450624. https://doi.org/10.2307/4450624 